\title{
Breakdown products of fibrin and fibrinogen: molecular mechanisms and clinical implications
}

\author{
PJ GAFFNEY
}

From the National Institute for Biological Standards and Control, Holly Hill, Hampstead, London

The major terminal enzymatic expression of the fibrinolytic cascade system in vivo is plasmin. This enzyme is presumed to interact with fibrinogen and fibrin in vivo and is arguably man's major defence against the deposition of fibrin, an important component in the general hazard of thrombosis.

Our understanding of the molecular details of plasmin interactions with fibrinogen and fibrin has been established over the past 10 years. As a part of the overall strategy to elaborate the primary sequence of human fibrinogen the precise locations of the peptide bonds ruptured by plasmin at various stages in the degradation process have been reported. Such precise data are not available as yet for fibrinplasmin interactions. I shall discuss the major aspects of fibrinogen-plasmin interactions only briefly since these have been recently reviewed by, among others, Mosesson and Finlayson ${ }^{1}$ and Gaffney.2 I shall deal with the current knowledge of fibrin-plasmin interactions in more detail since new and exciting data concerning fibrin fragmentation, which may have significance in vivo, have recently emerged.

In this review I am supposing that the major breakdown products of fibrinogen and fibrin (FDPs) are mediated by plasmin in vivo. Thus the in-vitro experiments conducted in many laboratories over the years have concentrated on plasmin-mediated interactions. It is, however, well to point out that the interactions between fibrinogen and a number of other proteolytic enzymes have also been studied in vitro (for review see Latallo et al. ${ }^{3}$ ). Indeed, Moroz and Gilmore 4 thought that a variety of proteolytic enzymes of cellular origin may play a far more important part in the lysis of established thrombin in vivo than does plasmin. Nevertheless, there is little doubt that plasmin interacts with forming fibrin, attempting to prevent its deposition on the vessel wall. I shall expand this last opinion when discussing plasmin-fibrin interactions and their significance in vivo.

\section{Fibrin-plasmin breakdown products}

FRAGMENTATION MECHANISMS

Investigations into molecular fragmentation began in 1945 when Walter Seegers, present-day doyen of haemostasis research workers, showed that plasmindigested fibrinogen consisted of two major electrophoretic fragments which were called $\alpha$-fibrinogen and $\beta$-fibrinogen. ${ }^{5}$ In the first detailed examination of the fragments obtained from the interaction of fibrinogen with plasmin Nussenzweig et al. ${ }^{6}$ distinguished five major fractions by ion-exchange chromatography. They called these A, B, C, D, and $E$, and they described fragments $D$ and $E$ as plasminresistant or terminal core fragments with molecular weights of about 83000 and 37000 respectively. Fragments D and E were $50 \%$ and $20 \%$ by weight of the original fibrinogen molecule, ${ }^{7}$ represented immunologically distinct regions of fibrinogen, ${ }^{8}$ and are synonymous with the $\alpha$ - and $\beta$-fibrinogens of Seegers. ${ }^{5}$ Nussenzweig et al. $^{6}$ also observed intermediate plasmin-labile degradation products of fibrinogen, subsequently named by other workers $\mathrm{X}, \mathrm{Y}$ fragments. ${ }^{9}$

Examination of the polypeptide compositions of the various fragments derived from plasminfibrinogen interactions in conjunction with carbohydrate staining and thrombin susceptibility of their composite peptide chains has allowed a number of schemes of fibrinogen fragmentation ${ }^{10-14}$ which have a number of common features. Fig. 1 shows the major features of a sequence of digestive reactions and products formed with which most of these workers would now agree. The conservation of both the $\mathrm{NH}_{2}$-terminal amino-acids ${ }^{16} 17$ of the $\mathrm{A} \alpha$-chains suggests that the initial lysis of the $A \alpha$-chain takes place at their carboxy ends. Peptides of about 40000 molecular weight are released rather rapidly, leaving the $\mathrm{NH}_{2}$-terminal remnant of the $\mathrm{A} \alpha$-chain disulphide bonded to intact $\mathrm{B} \beta$ and $\gamma$ chains. The next reaction involves the removal of peptides 


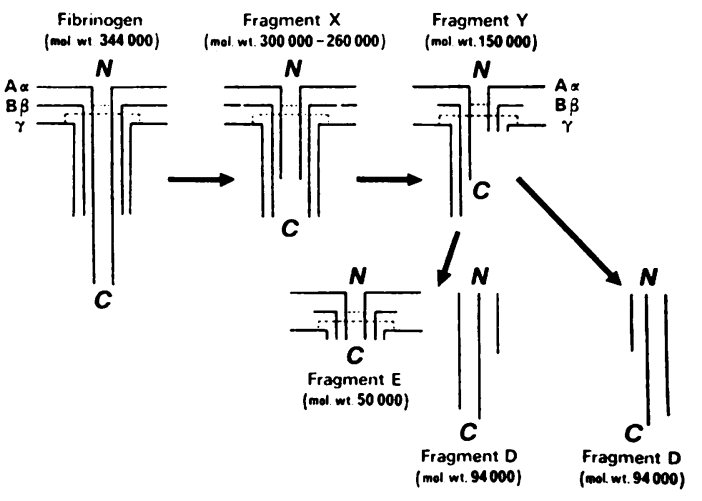

Fig. 1 Diagram of plasmin-mediated conversion of fibrinogen $(F g)$ to its core fragments $D$ and $E$, showing intermediate fragments $X$ and $Y$.

The scheme is constructed so that the polypeptide chain origin of the constituent subunits of each fragment can be related to those of the preceding fragment and in turn to chains of the originating fibrinogen molecule $(A \alpha, B \beta$, and $\gamma)$. The amino $(N)$ - and carboxy $(C)-$ terminal ends of the fragments are shown to aid in visualising the site of structural breakdown more easily. $M W s\left(\times 10^{-3}\right)$ are shown in parentheses after each fragment designation. The range of $M W$ s of fragment $X$ depends on the amount of peptide material removed from the carboxy ends of the A $\alpha$-chains and whether the peptides have been hydrolysed from the aminoterminal ends of the $B \beta$ chains. Other details of this asymmetrical scheme of fibrinogen fragmentation are in the text. (Taken from Gaffney ${ }^{15}$; reproduced by kind permission of the Editor and Publishers of Haemostasis.)

(MW 6000) from the $\mathrm{NH}_{2}$-terminal ends of the $\mathrm{B} \beta$-chains ${ }^{1217-21}$ followed by the asymmetric splitting of the three chains at one side of the partly degraded dimer. These latter cleavages release fragment $\mathbf{D}$, and thus all lysis points must be adjacent to the $\mathrm{NH}_{2}$-terminal ends of all three chains, allowing that fragment $D$ is located near the carboxy end of each fibrinogen subunit. ${ }^{22}$ The remaining major fragment is called $Y,{ }^{93}$ and the subsequent lysis of its $A \alpha$, $\mathbf{B} \beta$, and $\gamma$ chain remnants converts fragment $\mathbf{Y}$ to fragments D and E. Evidently the terminal or 'core' fragments $D$ are made up of three polypeptide chain remnants of the $\mathrm{A} \alpha, \mathrm{B} \beta$, and $\gamma$ chains of fibrinogen while fragment $\mathrm{E}$ is a dimer containing disulphide bonded subunits, each subunit being made up of three polypeptide chains.

This scheme of degradation owes much to the original scheme of Marder et al. ${ }^{23}$ in which they suggested an asymmetrical cleavage of the fibrinogen dimer by plasmin. This type of digestive splitting may suggest that the two subunits of this fibrinogen dimer differ discretely in primary structure or else that the sequential release of the $D$ fragments from each subunit of the fibrinogen dimer is random. Further support has been provided for the asymmetrical mode of fibrinogen fragmentation and the release of $\mathrm{D}$ as a monomeric fragment by the finding that the $\mathrm{NH}_{2}$-terminal amino-acids of fibrinogen, $\mathrm{X}, \mathrm{Y}$, and $\mathrm{E}$ are essentially alanine and tyrosine while those of fragment $\mathrm{D}$ are aspartic, methionine, and valine. This locates the $D$ fragment at the carboxy terminal half of the fibrinogen subunit. Subsequently, it has been shown ${ }^{24}$ that fragment $Y$ contains 2 mol fibrinopeptide $A$ and a set each of heavy and light polypeptide chains. These latter data seem to have confirmed beyond doubt the asymmetric scheme of fibrinogen degradation by plasmin shown in Fig. 1.

\section{Fibrin-plasmin interactions}

The thrombin-mediated reactions which accompany the conversion of fibrinogen to various types of fibrin must first be outlined.

Fibrin formation is initiated by the cleavage of the fibrinopeptides $A$ and $B$ from fibrinogen ( $\left.A \alpha_{2} B \beta_{2} \gamma_{2}\right)$ by thrombin. ${ }^{25}$ The resultant fibrin monomer $\left(\alpha_{2} \beta_{2 \gamma_{2}}\right)$ can polymerise to non-stabilised polymers $\left(\alpha_{2} \beta_{2} \gamma_{2}\right) p$, which can subsequently be cross-linked in the presence of calcium and factor XIII (activated by thrombin) to form stabilised fibrin. ${ }^{26}$ The glutamyl-lysyl ${ }^{27} 28$ cross-links form rapidly between the $\gamma$ chains to form $\gamma$ dimers $(\gamma-\gamma)$ while the $\alpha$ chains cross-link more slowly to form $\alpha$ chain polymers $\left(\alpha^{p}\right)$ of a molecular weight in excess of $400000 .^{29}$ It seems that the $\gamma$ chain cross-links can form in fibrin when it is still in the soluble state in plasma ${ }^{21}$ and the incorporation of $\alpha$ chain cross-links seems to depend on the $\gamma$ chains being already in situ.

The importance of these cross-links in fibrin and whether they have the opportunity of forming in vivo will be discussed later. For the moment it is sufficient to indicate that an understanding is required of the interactions of plasmin with at least three major forms of fibrin: (1) non-cross-linked fibrin (NXLFN); (2) partly $(\gamma-\gamma)$ cross-linked fibrin (PXL-FN); and (3) totally ( $\alpha$ chains and $\gamma$ chains cross-linked) cross-linked fibrin (TXL-FN). Part of the diagram in Fig. 2 shows three fibrin molecules with the approximate locations of the factor XIII cross-links. The $\alpha$ chain cross-links have been placed near the carboxy termini for ease of presentation, whereas we now know that they are located at least 20000 MW from the carboxy ends of the $\alpha$ chains. ${ }^{31}$

\section{NON-CROSS-LINKED FIBRIN (NXL-FN)}

From a chemical viewpoint it is reasonable to regard NXL-FN as being quite similar to fibrinogen, since its chemical structure lacks only fibrinopeptides 


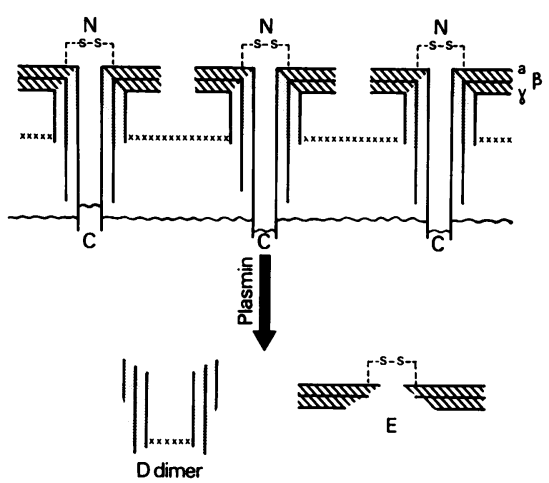

Fig. 2 Schematic representation of polypeptide chains of cross-linked human fibrin and its plasmin-mediated degradation products $D$ dimer and $E$.

The cross-linked $\gamma$-chain dimers, the cross-linked $\alpha$-chain polymers, and the uncross-linked $\beta$-chains, are here related to each other schematically. All the cross-links are shown for schematic convenience close to the carboxy $(C)$ ends of the respective chains.

While this localisation is true for the $\gamma$-chain cross-links the $\alpha$-chain cross-links are at least $20000 \mathrm{MW}$ distance from the C-terminal end. Disulphide $(S-S)$ bonds (not shown here except for one $S-S$ bond joining the two amino (N)-terminal ends of each fibrin molecule) link the individual polypeptide chains $(\alpha, \beta, \gamma)$ of each fibrin molecule. Non-covalent bonds also hold the units of the fibrin polymer together but are omitted for the sake of simplicity. The location of the antigenically distinct sections of fibrin $D$ and $E$ are shown in each of three fibrin molecules of the fibrin polymer. Plasmic digestion yields a fragment $D$ which is cross-linked to another $D$ fragment from an adjacent fibrin molecule. This type is called $D$ dimer. Fragment $E$ and a variety of cross-linked and uncross-linked peptides (not shown) are also released during the lysis of cross-linked fibrin. The lengths of the lines representing the polypeptide chains are not proportional to their MWs. (Taken from Gaffney et al..$^{30}$; reproduced by kind permission of the Editor, Cardiovascular Research.)

A and $\mathbf{B}$ while conformational differences are due to aggregation during the clotting process. Thus, not surprisingly, the digestion of NXL-FN by plasmin yields an array of fragments similar in molecular weight to those observed for fibrinogen. ${ }^{32-34}$ The susceptibility to plasmin of the individual chains in the intact NXL-FN is the same as that observed for fibrinogen. ${ }^{35}$ Obviously, of course, $X, Y$, and $E$ from fibrinogen will at least differ from those fragments obtained from NXL-FN in that the former contain fibrinopeptide $\mathrm{A}$ while the latter do not. Other discrete structural differences between the respective fragments from fibrinogen and NXL-FN exist which can be detected by radioimmunoassay using appropriate antisera. ${ }^{36} 37$
CROSS-LINKED FIBRIN (XL-FN)

The interaction of plasmin with XL-FN will deal with both PXL-FN and TXL-FN together since the spectrum of fragments obtained from both forms of fibrin is quite similar ${ }^{38}$ It would seem that the major property endowed on fibrin by $\alpha$ chain cross-linkage is resistance to lysis by plasmin..$^{39} 40$ Information about the fragments yielded on lysis of XL-FN by plasmin has slowly unfolded. In 1973 XL-FN digests were shown to contain a major distinct fragment not found in fibrinogen digests. ${ }^{33} 4142$ This was called D dimer ${ }^{32}$ since it was composed of two D domains from adjacent fibrin molecules, joined by their cross-linked $\gamma$ chain remmants. ${ }^{41}$

A scheme showing the origin of the $\mathrm{D}$ dimer and $\mathrm{E}$ fragments from XL-FN is shown in Fig. 2. Later it was found that some D dimer and E molecules could be present in a digestion mixture as an electrophoretically stable but non-covalent complex known as the $\mathrm{D}$ dimer-E complex. ${ }^{384344}$ Subsequent experiments, in which the lysis of XL-FN was performed in serum or plasma, indicated that all the $\mathrm{D}$ dimer and $\mathrm{E}$ molecules were present as a stoichometrically satisfied complex, having the general formula (D-D)E. 4546

The schematic representation of the $D$ dimer-E complex (Fig. 3) indicates that each domain originates in a distinct fibrin molecule. More important is that the $\mathrm{D}$ dimer-E complex must contain the major polymerisation sites of fibrin located in the $\mathrm{D}$ and $\mathrm{E}$ domains. ${ }^{47}$ Olexa and Budzynski ${ }^{48}$ showed that one mole of $D$ dimer and one mole of $E$ form one mole of the complex and that conversion of the complex to free $\mathrm{D}$ dimer and $\mathrm{E}$ fragments is mediated by digestion of the $\mathrm{E}$ domain. The $\mathrm{D}$ dimer- $\mathrm{E}$ complex can dissociate in $8 \mathrm{M}$ urea and reforms after removal of the detergent. ${ }^{45}$ Specific ant:genic markers have been recognised in $\mathrm{D}$ dimer and the $\mathrm{D}$ dimer-E complex ${ }^{49}$ but since these crosslink-related markers represent only a small proportion of the total antigenicity of the $\mathbf{D}$ dimer fragment it is difficult to devise a useful test for these fragments in vivo.

In view of the evidence that the $D$ dimer-E complex is a major product of cross-linked fibrin lysis in vivo, ${ }^{5051}$ a variety of lytic regimens was investigated to determine whether this complex was the earliest soluble expression of XL-fibrin lysis. It transpired that besides the $\mathrm{D}$ dimer-E complex higher molecular weight complexes are released from XL-FN which have been classified as crosslinked fragment X-oligomers, Y-D, and $\mathrm{D}$ dimer complexes. Schematic structures of these fibrin degradation products (Fig. 4) were elucidated from sodium dodecyl sulphate polyacrylamide gel electrophoresis, carbohydrate staining, and 125I-label distribution. ${ }^{52}$ Thus it would seem that cross-linked 


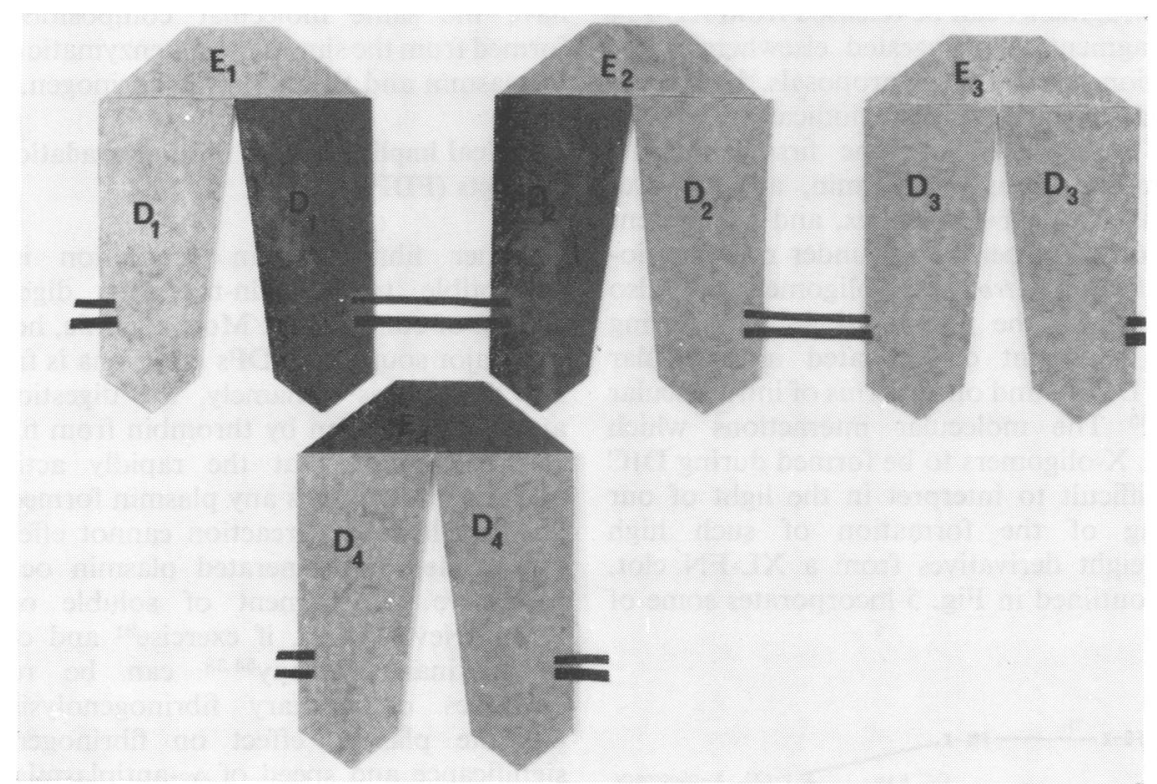

Fig. 3 Schematic view of probable fibrin subunit origins of the three component parts of the $D$ dimer-E complex. The intermolecular $\gamma$ chain cross-links are shown as solid lines, while the shaded area shows that two $D$ fragments from adjacent fibrin subunits and an E fragment from another fibrin subunit are associated in the $D$ dimer-E complex $\left(D_{1}-D_{2}\right) E_{4}$. The suggestion is inherent that the COOH-terminal ends of the $\gamma$ chains of fibrin which contain the cross-linking residues are also involved in the binding of one $E$ fragment between the two D fragments.

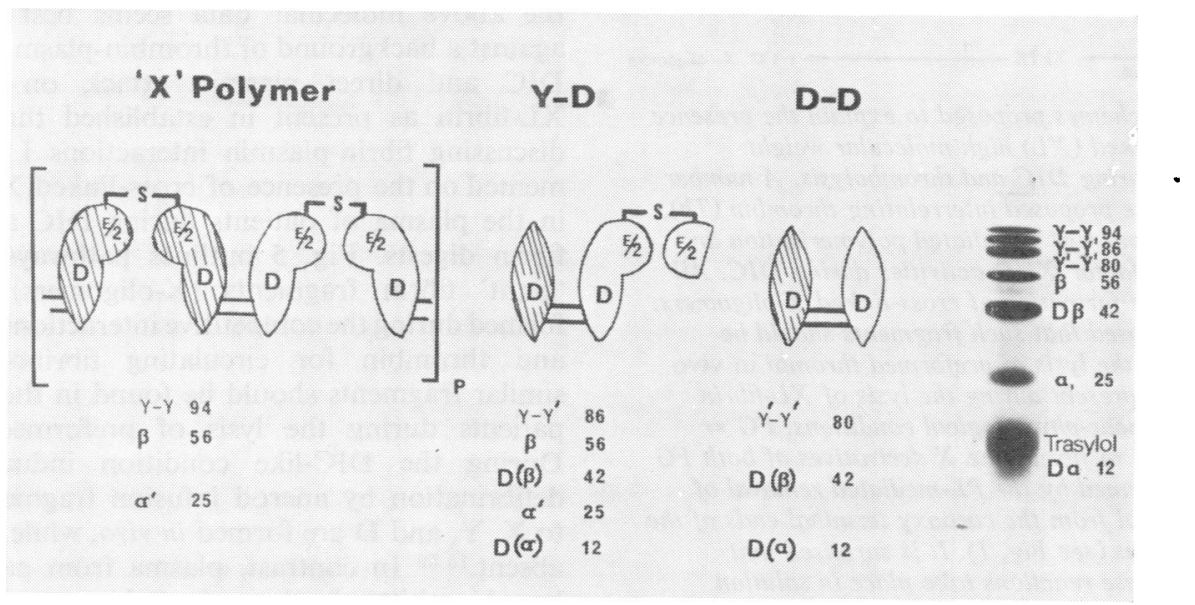

Fig. 4 Diagrammatic presentations of the various cross-linked fragments found in the plasmin-mediated lysates of cross-linked fibrin. The polypeptide chain components of the various cross-linked classes of fragments are shown below the relevant diagram.

The polyacrylamide gel separation of the various polypeptide chains which comprise the combined high molecular weight cross-linked fragments is shown to the right of the diagram. The nomenclature used for the chains has been described elsewhere. ${ }^{22}$ There is evidence to suggest that all these cross-linked fragments can remain associated with each other, possibly through the D dimer-E polymerisation site, and separate only when subjected to detergent treatment before electrophoresis. 
$\mathrm{X}, \mathrm{Y}$, and $\mathrm{D}$ fragments can be released from XL-FN as soluble fragments, as suggested elsewhere ${ }^{53}$ and in contradiction to our earlier proposals. ${ }^{54}$

Our unpublished data also indicate that the cross-linked $\mathrm{X}$-oligomers are the first fragments derived from XL-fibrin by plasmin, are the precursors of the $\mathrm{D}$ dimer-E complex, and are present during lysis of fibrin performed under near-physiological conditions in vitro. These oligomers have also been isolated from the plasma of patients during episodes of fulminant disseminated intravascular coagulation (DIC) ${ }^{54}$ and other forms of intravascular coagulation. ${ }^{55}$ The molecular interactions which allow the XL X-oligomers to be formed during DIC are rather difficult to interpret in the light of our understanding of the formation of such high molecular weight derivatives from a XL-FN clot. The scheme outlined in Fig. 5 incorporates some of

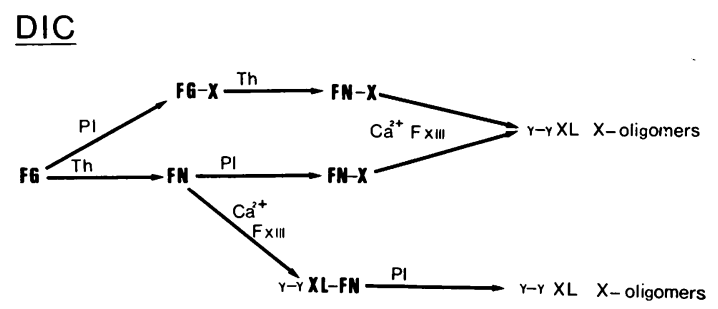

Thrombolysis

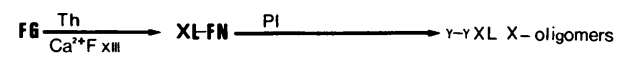

Fig. 5 Two schemes proposed to explain the presence of the cross-linked $(X L)$ high molecular weight $X$-oligomers during DIC and thrombolysis. A number of pathways are proposed interrelating thrombin (Th), plasmin $(\mathrm{Pl})$, and $\mathrm{Ca}^{++}-$mediated polymerisation and cross-linking (factor XIII) activities during DIC. All could allow the formation of cross-linked $X$-oligomers. It is also proposed that such fragments should be formed during the lysis of preformed thrombi in vivo since they are present during the lysis of XL-fibrin in vivo under near-physiological conditions. $F G=$ fibrinogen; $F N=$ fibrin; the $X$ derivatives of both $F G$ and FN are formed by the PL-mediated removal of peptide material from the carboxy terminal ends of the $A \alpha$ and $\alpha$ chains (see Fig. 1). It is surmised that during DIC these reactions take place in solution without deposition of fibrin.

our thoughts with those of Graeff and his colleagues $^{55}$ and suggests that the XL X-oligomers could occur by a number of pathways which involve the competition of plasmin and thrombin for fibrinogen and fibrin during DIC. It seems that the earliest fragments formed during fibrin clot lysis have the same molecular composition as those formed from the simultaneous enzymatic competition of plasmin and thrombin for fibrinogen.

\section{Practical implications of fibrin degradation products (FDPs)}

Whether fibrinogen in circulation is normally susceptible to plasmin-mediated digestion is a subject of controversy. Most concede, however, that the major source of FDPs in plasma is from secondary fibrinolysis ${ }^{56}$-namely, the digestion of fibrin after its generation by thrombin from fibrinogen. It can be argued that the rapidly acting $\alpha_{2}$-antiplasmin ${ }^{57}$ neutralises any plasmin formed in circulation, while such a reaction cannot effectively take place when the generated plasmin occurs in the protective environment of soluble or insoluble fibrin. Nevertheless, if exercise ${ }^{31}$ and conventional streptokinase therapy 5458 can be regarded as examples of primary fibrinogenolysis in their ultimate plasmic effect on fibrinogen then the significance and speed of $\alpha_{2}$-antiplasmin activity in blood may need reappraisal. These notions are in part supported by the fact that streptokinase induces a greater reduction in circulating $\alpha_{2}$-antiplasmin than that observed during ancrod infusion, which induces secondary and far more effective fibrinolysis (Gaffney, unpublished data).

Thus our consideration of the practical value of the above molecular data seems best considered against a background of thrombin-plasmin mediated DIC and direct plasmin attack on preformed XL-fibrin as present in established thrombi. ${ }^{30}$ In discussing fibrin-plasmin interactions I have commented on the presence of cross-linked X-oligomers in the plasma of patients during DIC and in XLfibrin digests. Fig. 5 outlines pathways by which 'giant' fibrin fragments (X-oligomers) could be formed during the competitive interactions of plasmin and thrombin for circulating fibrinogen, while similar fragments should be found in the plasma of patients during the lysis of preformed thrombi. During the DIC-like condition induced during defibrination by ancrod infusion fragments similar to $\mathrm{X}, \mathrm{Y}$, and $\mathrm{D}$ are formed in vivo, while $\mathrm{D}$ dimer is absent. ${ }^{1559}$ In contrast, plasma from patients who have been bitten by the snake Echis carinatus contains significant amounts of $\mathbf{D}$ dimer. ${ }^{60}$ The conversion of prothrombin to thrombin by the snake venom allows the formation of factor XIII-mediated cross-linked fibrin and its subsequent digestion to cross-linked $\mathrm{D}$ fragments. The latter clinical condition bears some of the hallmarks of advanced DIC in contradistinction to the benign condition of the patient during defibrination with ancrod, a 
procoagulant from the venom of Agkistrodon rhodostoma. Coagulation disorders (mostly DIC) in obstetric patients were accompanied by the presence in the plasma of large fibrin fragments similar to the $\mathrm{X}$-oligomers described above in the XL-fibrin digests, ${ }^{55}$ while cases of fulminant DIC had crosslinked X-oligomers and the D dimer-E complex in the plasma. ${ }^{51}$

Emerging from case reports such as those referred to above is a hypothesis (Fig. 6) which echoes and

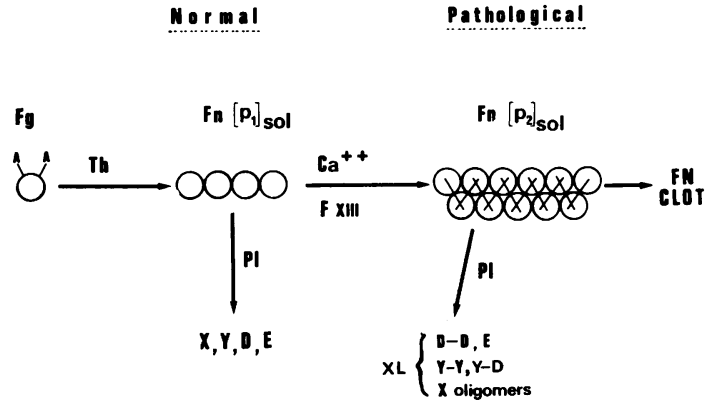

Fig. 6 Diagram outlining possible sequences of events in vivo during the hypercoagulable or prethrombotic state.

After the removal of fibrinopeptide $A$ from fibrinogen $(F g)$ by thrombin $(T h)$ soluble fibrin polymers $\left(F n_{p}\right)$ form which can be converted by plasmin $(\mathrm{Pl})$ to fibrin degradation products $(X, Y, D, E)$. Such reactions are thought to occur at a local level regularly in healthy subjects. Should either the available Th production be excessive or the level of plasmin depressed large fibrin polymers $\left(F_{n} n_{2}\right)$ may form and cross-link under the influence of $\mathrm{Ca}^{++}$and factor XIII. These cross-linked fibrin polymers can act as a focus for clot formation or can be digested by plasmin to generate cross-linked fragments-for example, $(D-D) E, Y-Y, Y-D$, and $X$-oligomers. The diagram proposes that the detection in plasma of XL fibrin polymers or their plasminmediated fragments defines a 'pathological' prethrombotic state.

extends the thoughts of Astrup ${ }^{61}$ about coagulation and fibrinolysis being systemically balanced to maintain blood fluidity and competent haemostasis. It is now more probable that this balance operates at a local rather than a systemic level. The amount of available thrombin depends on the amount of circulating inhibitors (notably At III) which operate at various points in the coagulation cascade. The amount of plasmin available for fibrin digestion seems to be independent of the concentrations of fibrinolytic inhibitors (notably the fast-acting $\alpha_{2}$-antiplasmin) since plasminogen seems to be protected from inhibition by the forming fibrin polymers.
The hypothesis presented in Fig. 6 suggests that the composition of the fragments formed during the competition between thrombin and plasmin should shed light on the severity of the so-called hypercoagulable state in the patient's blood. The presence of non-cross-linked fragments would suggest that the fibrinolytic system was rapidly digesting forming fibrin, whereas large cross-linked fragments (X-oligomers) together with cross-linked $\mathrm{D}$ dimer-E, $Y-Y$, and Y-D complexes would suggest that the hypercoagulability was quite severe in that the fibrinolytic system was being overpowered and barely coping with the fibrin polymerisation process. The latter instance could be detected by sensitive assays for these high molecular weight cross-linked complexes, and such assays might be useful in highlighting the clinically-dangerous hypercoagulable or thrombotic state.

Similar concepts about the haemostatic balance during DIC have been outlined by others ${ }^{62}$ using assay procedures for fibrinopeptide $A$ and the $\mathrm{NH}_{2}$-terminal $\mathrm{B} \beta$ peptide (1-42) to determine the influence of thrombin and plasmin respectively. We believe, however, that the presence of the crosslinked fragments described above (and shown in Figs. 4 and 6) in a patient's plasma would indicate a more advanced state of hypercoagulability than would be detected by the tests suggested by Nossel and colleagues. ${ }^{63}$ Whereas these cross-linked fragments have been found in the plasma of patients during DIC, assays have yet to be developed of sufficient sensitivity to monitor their presence in plasma during thrombolytic therapy for thrombosis. To this end antisera have been raised in rabbits which contain conformational neo-antigens for the cross-linked X-oligomers. These antisera do not react with fibrinogen and their use is contemplated in radioimmunoassay to detect the advanced prethrombotic state and to clarify the role of fibrinolysis as man's major defence against thrombosis.

\section{References}

1 Mosesson MW, Finlayson JS. The search for the structure of fibrinogen. In: Spaet TH, ed. Progress in haemostasis and thrombosis, vol 3. New York: Grune and Stratton, 1976:61-107.

2 Gaffney PJ. The biochemistry of fibrinogen and fibrin degradation products. In: Ogston D, Bennett B, eds. Haemostasis, biochemistry, physiology, and pathology. New York: John Wiley, 1977:105-68.

${ }^{3}$ Latallo ZS, Teisseyre E, Ardelt W, Wegrzynowitz Z, Kopeć M. Fibrino(geno)lysis by enzymes other than plasmin. In: Gaffney PJ, Balkov-Ulutin S, eds. Fibrinolysis: current fundamental and clinical 
concepts. London: Academic Press, 1978:129-36.

${ }^{4}$ Moroz LA, Gilmore NJ. Fibrinolysis in normal plasma and blood; evidence for significant mechanisms independent of the plasminogen-plasmin system. Blood 1976;48:531-45.

${ }^{5}$ Seegers WH, Nieft ML, Vandenbelt JM. Decomposition products of fibrinogen and fibrin. Arch Biochem 1945;7:15-9.

${ }^{6}$ Nussenzweig V, Seligmann M, Pelmont J, Grabar P. Les produits de degradation du fibrinogene humain par la plasmine. I. Separation et proprietes physicochimiques. Ann Inst Pasteur (Paris) 1961;100:377-89.

7 Jamieson GA, Gaffney PJ. Nature of the high molecular weight fraction of fibrinolytic digests of human fibrinogen. Biochim Biophys Acta 1968;154:96-109.

${ }^{8}$ Marder VJ. Identification and purification of fibrinogen degradation products produced by plasmin: considerations on the structure of fibrinogen. Scand J Haematol (Suppl) 1971 ;13:21-36.

${ }^{9}$ Larrieu MJ, Marder VJ, Inceman S. Effects of fibrinogen degradation products on platelets and coagulation. Thromb Diath Haemorrh 1966;20 (Suppl): 215-26.

${ }^{10}$ Mills DA. A molecular model for the proteolysis of human fibrinogen by plasmin. Biochim Biophys Acta 1972;263:619-30.

11 Mills DA, Karpatkin S. The initial macromolecular derivatives of human fibrinogen produced by plasmin. Biochim Biophys Acta 1972;271:163-73.

${ }^{12}$ Mosesson MW, Finlayson JS, Galanakis DK. The essential covalent structure of human fibrinogen evinced by analysis of derivatives formed during plasmic hydrolysis. J Biol Chem 1973;248:7913-29.

${ }^{13}$ Furlan M, Beck EA. Plasmin degradation of human fibrinogen. 1. Structural characterisation of degradation products. Biochim Biophys Acta 1972;263: 631-44.

${ }^{14}$ Pizzo SV, Schwartz ML, Hill RL, McKee PA. The effect of plasmin on the subunit structure of human fibrinogen. J Biol Chem 1972;247:636-45.

15 Gaffney PJ. Fibrin(ogen)interactions with plasmin. Haemostasis 1977;6:2-25.

${ }^{16}$ Sherman LA, Mosesson MW, Sherry S. Isolation and characterization of the clottable low molecular weight fibrinogen derived by limited plasmin hydrolysis of human fraction I-4. Biochemistry 1969; 8:1515-23.

${ }^{17}$ Mosesson MW, Finlayson JS, Umfleet RA, Galanakis DK. Human fibrinogen heterogenetics. I. Structural and related studies of plasma fibrinogens which are high solubility catabolic intermediates. $\mathrm{J} \mathrm{Biol} \mathrm{Chem}$ 1972;247:5210-9.

${ }^{18}$ Shainoff JR, Lahiri B, Bumpus FM. Ultracentrifuge studies on the reaction between thrombin and plasminized fibrinogen. Thromb Diath Haemorrh (Suppl) 1970;39:203-17.

${ }^{19}$ Lahiri B, Bumpus FM, Shainoff JR. Fate of the fibrinopeptides in the reaction between human plasmin and fibrinogen. Fed Prac 1970;29:648.

${ }^{20}$ Budzynski AZ, Marder VJ, Shainoff JR. The content of fibrinopeptides $\mathbf{A}$ and $\mathbf{B}$ in purified degradation products of human fibrinogen. Fed Proc 1973;32:
313.

${ }^{21}$ Kierulf P. N-terminal analysis of fibrins from plasmin hydrolysed fibrinogen-evidence for lack of fibrinopeptide B. Thromb Res 1972;1:527-32.

${ }^{22}$ Gaffney PJ. Localisation of carbohydrate in the subunits of human fibrinogen and its plasmininduced fragments. Biochim Biophy Acta 1972;263: 453-8.

${ }^{23}$ Marder VJ, Shulman NR, Carroll WR. High molecular weight derivatives of human fibrinogen produced by plasmin. I. Physicochemical and immunological characterization. J Biol Chem 1969; 244:2111-9.

${ }^{24}$ Budzynski AZ, Marder VJ, Shainoff JR. Structure of plasmin degradation products of human fibrinogen. Fibrinopeptide and polypeptide chain analysis. J Biol Chem 1974;249:2294-302.

${ }^{25}$ Bettelheim FR, Bailey $\mathrm{K}$. The products of the action of thrombin on fibrinogen. Biochim Biophys Acta 1952;9:578-9.

${ }^{26}$ Lorand L, Konishi K. Activation of the fibrin stabilizing factor of plasma by thrombin. Arch Biochem Biophys 1964;105:58-67.

${ }^{27}$ Matacic S, Loewy AG. The identification of isopeptide cross-links in insoluble fibrin. Biochem Biophys Res Commun 1968;30:356-62.

${ }^{28}$ Pisano JJ, Finlayson JS, Peyton MP. Cross-link in fibrin polymerized by factor XIII; $\epsilon-(\gamma$-glutamyl $)$ lysine. Science 1968;160:892-3.

29 McKee PA, Mattock P, Hill RL. Subunit structure of human fibrinogen, soluble fibrin and cross-linked insoluble fibrin. Proc Natl Acad Sci USA 1970;66: 738-44.

${ }^{30}$ Gaffney PJ, Brasher M, Lord K, et al. Fibrin subunits in venous and arterial thromboembolism. Cardiovasc Res 1976;10:421-6.

${ }^{31}$ Finlayson JS, Mosesson MW. Crosslinking of $a$ chain remnants in human fibrin. Thromb Res 1973;2:46778.

${ }^{32}$ Gaffney PJ. Subunit relationships between fibrinogen and fibrin degradation products. Thromb Res 1973; 2:201-17.

${ }^{33}$ Pizzo SV, Schwartz ML, Hill RL, McKee PA. The effect of plasmin on the subunit structure of human fibrin. J Biol Chem 1973;248:4574-83.

${ }^{34}$ Slade CL, Pizzo SV, Taylor LM Jr, Steinman HM, McKee PA. Characterisation of fragment $E$ from fibrinogen and cross-linked fibrin. $J$ Biol Chem 1976;251 :1591-6.

${ }^{35}$ Gaffney PJ, Dobos P. A structural aspect of human fibrinogen suggested by its plasmin degradation. FEBS Lett 1971 ;15:13-6.

${ }^{36}$ Edgington TS, Plow EF. Conformational and structural modulation of the $\mathrm{NH}_{2}$-terminal regions of fibrinogen and fibrin associated with plasmin cleavage. J Biol Chem 1975;250:3393-8.

${ }^{37}$ Edgington TW, Plow E. Differentiation between fibrinogen and fibrin cleavage fragments based on immunochemical correlates of structure and clinical implications. In: Neri Serneri GG, Prentice CRM, eds. Haemostasis and thrombosis (Proceedings of the Serono Foundation Symposia, vol 15). London: 
Academic Press, 1979:27-43.

${ }^{38}$ Gaffney PJ, Lane DA, Brasher M. Soluble high molecular weight $\mathrm{E}$ fragments in the plasmininduced degradation products of crosslinked human fibrin. Clin Sci Mol Med 1975;49:149-56.

${ }^{39}$ Schwartz ML, Pizzo SV, Hill RL, McKee PA. The effect of fibrin-stabilizing factor on the subunit structure of human fibrin. J Clin Invest 1971;50: 1506-13.

${ }^{40}$ Gaffney PJ, Whitaker AN. Fibrin crosslinks and lysis rates. Thromb Res 1979;14:85-94.

41 Gaffney PJ, Brasher M. Subunit structure of the plasmin-induced degradation products of crosslinked fibrin. Biochim Biophys Acta 1973;295:308-13.

${ }^{42}$ Kopeć M, Teisseyre E, Dudek-Wojciechowska G, Kocewiak M, Pankiewicz A, Latallo ZS. Studies on the 'double D' fragment from stabilized bovine fibrin. Thromb Res 1973;2:283-91.

${ }^{43}$ Hudry-Clergeon G, Paturel L, Suscillon M. Identification d'un complexe (D-D) ...E dans les produits de dégradation de la fibrine bovine stabilisée par le factor XIII. Pathol Biol (Suppl) 1974;22:47-52.

${ }^{44}$ Gaffney PJ, Lane DA, Kakkar VV, Brasher M. Characterisation of a soluble D dimer-E complex in crosslinked fibrin digests. Thromb Res 1975;7: 89-99.

${ }^{45}$ Gaffney PJ. The relevance of the D dimer-E complex to the lysis of cross-linked fibrin. In: Davidson JF, Cěpélak V, Samama MM, Desnoyers PC, eds. Progress in chemical fibrinolysis and thrombolysis, vol 4. Edinburgh: Churchill Livingstone, 1979: 424-32.

${ }^{46}$ Gaffney PJ, Joe F. The lysis of crosslinked human fibrin by plasmin yields initially a single molecular complex, D dimer-E. Thromb Res 1979;15:673-97.

${ }^{47}$ Kudıyk BJ, Collen D, Woods KR, Blombäck B. Evidence for localization of polymerization sites in fibrinogen. J Biol Chem 1974;249:3322-5.

${ }^{48}$ Olexa SA, Budzynski AZ. Primary soluble plasmic degradation product of human cross-linked fibrin. Isolation and stoichiometry of the (DD)E complex. Biochemistry 1979;18:991-5.

${ }^{43}$ Budzynski AZ, Marder VJ, Parker ME, Shames P, Brizuela BS, Olexa SA. Antigenic markers on fragment DD, a unique plasmic derivative of human crosslinked fibrin. Blood 1979;54:794-804.

${ }^{50}$ Gaffney PJ. Distinction between fibrinogen and fibrin degradation products in plasma. Clin Chim Acta $1975 ; 65: 109-15$.

${ }^{51}$ Whitaker AN, Rowe EA, Masci PP, Gaffney PJ. Identification of $\mathrm{D}$ dimer-E complex in disseminated intravascular coagulation (DIC). Thromb Haemostas 1979;42:274.

${ }^{52}$ Gaffney PJ, Joe F. The D dimer-E complex and its aggregates in crosslinked fibrin digests. Thromb Haemostas 1979;42:427.

${ }^{53}$ Regañon E, Vila V, Aznar J. Identification of high molecular weight derivatives of plasmic digests of cross-linked human fibrin. Thromb Haemostas 1978;40:368-76.

${ }^{54}$ Gaffney PJ. The molecular and functional condition of plasma fibrinogen during thrombolytic therapy with streptokinase. Thromb Res 1973;2:105-14.

${ }^{55}$ Graeff H, Hafter R, Bachmann L. Subunit and macromolecular structure of circulating fibrin from obstetric patients with intravascular coagulation. Thromb Res 1979;16:313-28.

${ }^{56}$ Nilsson IM, ed. Haemorrhagic and thrombotic disease. London: John Wiley, 1971:111-62.

${ }^{57}$ Collen D. Identification and some properties of a new fast-acting plasmin inhibitor in human plasma. Eur J Biochem 1976;69:209-16.

${ }^{58}$ Gaffney PJ, Chesterman CN, Allington MJ. Plasma fibrinogen and its fragments during streptokinase treatment. Br J Haematol 1974;26:285-93.

59 Prentice CRM, Edgar W, McNicol GP. Characterisation of fibrin degradation products in patients on ancrod therapy: comparison with fibrinogen derivatives produced by plasmin. $\mathrm{Br} \mathrm{J}$ Haematol 1974;27:77-87.

${ }^{60}$ Edgar W, Warrell MJ, Warrell DA, Prentice CRM. The structure of soluble fibrin complexes and fibrin degradation products after Echis carinatus bite. Br J Haematol 1980 (in press).

${ }^{61}$ Astrup T. The biological significance of fibrinolysis. Lancet 1956;2:565-8.

${ }^{62}$ Nossel HL, Canfield R, Kaplan K, et al. Fibrinogen $B \beta$ chain proteolysis by thrombin and plasmin-a regulatory mechanism in hemostasis and thrombosis. Thromb Haemostas 1979;42:427. 